This item was submitted to Loughborough's Research Repository by the author.

Items in Figshare are protected by copyright, with all rights reserved, unless otherwise indicated.

\title{
Magnetic flux quantum periodicity of the frequency of the on-chip detectable electromagnetic radiation from superconducting flux-flow-oscillators
}

PLEASE CITE THE PUBLISHED VERSION

https://doi.org/10.1063/5.0021970

PUBLISHER

AIP Publishing

VERSION

AM (Accepted Manuscript)

\section{PUBLISHER STATEMENT}

This article may be downloaded for personal use only. Any other use requires prior permission of the author and AIP Publishing. This article appeared in B. Chesca et al., Appl. Phys. Lett. 117, 142601 (2020) and may be found at https://doi.org/10.1063/5.0021970.

\section{LICENCE}

All Rights Reserved

\section{REPOSITORY RECORD}

Chesca, Boris, Daniel John, Marat Gaifullin, Jonathan Cox, Aidan Murphy, Sergey Saveliev, and Christopher Mellor. 2020. "Magnetic Flux Quantum Periodicity of the Frequency of the On-chip Detectable Electromagnetic Radiation from Superconducting Flux-flow-oscillators". Loughborough University. https://hdl.handle.net/2134/13061297.v1. 


\title{
Magnetic Flux Quantum Periodicity of the Frequency of the On-chip Detectable Electromagnetic Radiation from Superconducting Flux-flow- oscillators
}

\author{
Boris Chesca*1, Daniel John ${ }^{1}$, Marat Gaifullin ${ }^{1,3}$, \\ Jonathan Cox $^{1}$, Aidan Murphy ${ }^{1}$, Sergey Savel' ${ }^{1}{ }^{1}$, Christopher J. Mellor ${ }^{2}$ \\ ${ }^{1}$ Department of Physics, Loughborough University \\ Loughborough, LE11 3TU, United Kingdom \\ ${ }^{2}$ School of Physics and Astronomy, University of Nottingham \\ Nottingham, NG7 2RD, United Kingdom \\ ${ }^{3}$ SuperOx Japan LLC, Sagamihara, 252-0243, Japan
}

\author{
Corresponding author: \\ Dr. Boris Chesca \\ Physics Department \\ Loughborough University \\ Loughborough, \\ UK \\ Email: $\underline{\text { B.Chesca@lboro.ac.uk }}$ \\ Phone: $+44(0) 1509223985$
}

\begin{abstract}
Superconducting flux-flow-oscillators (FFO) based on unidirectional flow of magnetic vortices in a single-long Josephson junction (JJ) and operating at $4.2 \mathrm{~K}$ are key elements of sub-terahertz integrated-receivers used in radio-astronomy and atmospheric science. Here we report on the development of sub-terahertz FFO's based on parallel JJ-arrays made of $\mathrm{YBa}_{2} \mathrm{Cu}_{3} \mathrm{O}_{7-\delta}$ thin films. Sharp multiple flux-flow resonances were observed in the temperature range (77-89) $\mathrm{K}$ in asymmetric JJ-arrays suggesting they can operate as narrow-
\end{abstract}


band FFO in sub-terahertz integrated-receivers at more practical temperatures than $4.2 \mathrm{~K}$. We detected electromagnetic radiation (EM) emitted by symmetric JJ-arrays in the range (30-45) $\mathrm{K}$ using on-chip build superconducting detectors based on single JJs. For both asymmetric and symmetric JJ-arrays the frequency $f$ of the emitted radiation could be tuned continuously by an applied magnetic flux $\Phi$ with a one-flux-quantum $\Phi_{0}$ periodicity. Remarkably, since $f$ can be tuned continuously there are no gaps in the frequency range of the emitted EM. The fundamental $\Phi_{0}$-periodicity of $f(\Phi)$ is similar in nature to a SQUID's voltage response $V(\Phi)$ and, consequently, using high-performance magnetic flux-to-field conversion and read-out techniques a sensitive field-to-frequency magnetometer can be developed. Incorporated into non-accessible micro/nanostructures as a magnetic sensor it would allow precise measurements of magnetic-fields from a distance, without the need to measure it locally as the radiation is detected remotely.

Flux-flow-oscillators $(\mathrm{FFO})^{1-7}$ based on unidirectional flow of magnetic vortices in a single-long Josephson junction $(\mathrm{JJ})^{1,4-7}$ or two-dimensional arrays of $\mathrm{JJs}^{2-3}$ are natural, tunable, emitters/receivers of electromagnetic (EM) radiation. FFO's operating at $4.2 \mathrm{~K}$ have been implemented to develop ultra-sensitive superconducting sub-terahertz integratedreceivers routinely used in radio-astronomical research ${ }^{8}$ or atmospheric science projects ${ }^{9,10}$. Here we report on the development of sub-terahertz FFO's based on parallel JJ-arrays made of $\mathrm{YBa}_{2} \mathrm{Cu}_{3} \mathrm{O}_{7-\delta}(\mathrm{YBCO})$ thin films and operating at more practical temperature than $4.2 \mathrm{~K}$ in the range (30-89) K. We detected electromagnetic radiation (EM) emitted by symmetric JJarrays in the range (30-45) $\mathrm{K}$ by observing the induced Shapiro steps ${ }^{11}$ on the dc currentvoltage characteristics (IVCs) of the $J J$ detectors made of single JJs. The frequency $f$ of the emitted radiation could be tuned continuously by an applied magnetic field B (or, equivalently, magnetic flux $\Phi=$ BAloop $)$ with a periodicity of one flux quantum $\Phi_{0}$ per superconducting loop area $\mathrm{A}_{\text {loop }}$ separating each pair of JJs. The $\Phi_{0}$-periodicity of $f(\Phi)$ in $J J$ arrays is a striking advantage to the linear dependence $f(\Phi)$ of flux-flow resonances in single

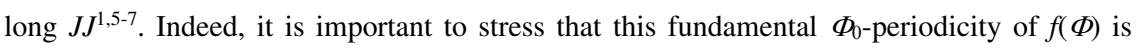
similar in nature to the $\Phi_{0}$-periodicity ${ }^{12-13}$ of the SQUID's voltage response $V(\Phi)$. Considering this similarity one can make use of the high-performance magnetic flux-to-field conversion and read-out techniques developed for SQUIDs ${ }^{14}$ in order to build a sensitive field-to-frequency magnetometer. This is useful considering that, despite their unique field 
sensitivity, SQUIDs-magnetometers cannot be used remotely while, in some applications, they measure the magnetic field $B$ at a certain distance from the source. Those readings are unavoidable approximate. Solving complex inverse problems with non-unique solutions for the current's distributions consistent with measurements of $B$ is often ambiguous. A significantly more accurate method in these situations will be to "sense" $B$ locally and measure it remotely from a short distance. In other applications it is required to measure $B$ inside non-accessible nano/micro-structures. A SQUID cannot be used as it requires a direct wire connection to the read-out electronics. For all these reasons the development of a highly sensitive, remote magnetometer whose sensor is located in close proximity to the source of B to be measured would open complementary avenues in many cutting-edge research areas. Interestingly, a related concept made earlier on $^{15}$ to develop a flux detector by tuning the radiation frequency of a SQUID with an applied magnetic field could not materialize in practice as in this case the frequency could not be tuned by more than $15 \%$. By comparison, JJ-arrays operated as flux-flow oscillators are far more superior with an increased EMradiation emission power and the ability to tune its frequency over a much wider range with an applied B.

It is useful to briefly clarify the physical origin of EM-radiation emitted due to the flux-flow resonances in $J J$-arrays ${ }^{16-21}$. When a $B$ field is applied perpendicular to a planar one-dimensional $J J$-array, magnetic vortices will enter the array in a form of Josephson vortices. The bias current, $I$, flowing across the array produces a Lorentz force which drives the Josephson vortices unidirectionally, forming a lattice of vortices moving with a certain speed. This is accompanied by an emission of EM-waves that propagate along the array. When the vortex spacing is commensurate with the wavelength of emitted EM-waves, resonant modes occur. This can be viewed as the phase-locking condition of the vortex velocity and the phase velocity of one of the self-induced EM-modes. The experimental signature of such phase locking between a train of propagating vortices and their induced EM-radiation in a $J J$-array is a series of flux-flow resonances $m=1-4$ in the dc IVCs. On a resonant current step, moving vortices couple to their induced linear waves. The emission of the EM-radiation is maximum at the resonance and is typically in the region of hundreds of $\mathrm{nW}$. Such flux-flow resonances have previously been observed in $J J$-arrays made of lowtemperature superconductors (LTS) operating at $4.2 \mathrm{~K}^{16-20}$, as well as, high-temperature superconductors (HTS) operating in the range (77-89) $\mathrm{K}^{21}$. Similarly, flux-flow induced 
enhanced EM-radiation has recently been measured ${ }^{22}$ in the absence of a magnetic field in LTS JJ-arrays operating at $4.2 \mathrm{~K}$.

The $J J$-arrays were fabricated by depositing high quality epitaxial, $100 \mathrm{~nm}$ thick $c$ axis oriented $\mathrm{YBa}_{2} \mathrm{Cu}_{3} \mathrm{O}_{7-\delta}$ (YBCO) films on $10 \times 10 \mathrm{~mm}^{2}, 24^{\circ}$ symmetric [001] tilt $\mathrm{SrTiO}_{3}$ bicrystals by pulsed laser deposition. Medium-underdoped YBCO films with a critical temperature $T_{c}=49 \mathrm{~K}$, as well as, optimally doped YBCO films with $T_{c}=92 \mathrm{~K}$, were subsequently patterned by optical lithography and etched by an Ar ion beam to form either symmetric $100 \mathrm{JJ}$-arrays (see Fig. 1) or asymmetric $440 \mathrm{JJ}$-arrays, respectively. Within the symmetric $100 \mathrm{JJ}$-arrays all $J J_{S}$ are $3 \mu \mathrm{m}$ wide and are separated by superconducting rectangular loops of identical area $\mathrm{A}_{\mathrm{loop}}=3 \times 15 \mu^{2}$. The asymmetric $440 \mathrm{JJ}$-arrays consist of 22 identical sets of $20 \mathrm{JJ}$ each separated by superconducting rectangular loops of nonidentical areas (widths) ranging from $A_{\text {loop }}=3 \times 8 \mu \mathrm{m}^{2}$ to $3 \times 13 \mu \mathrm{m}^{2}$. A magnetic field $B$ is applied perpendicular to the planar array structures via a control current $I_{B}$ through an inductively coupled coil. Consequently, an external magnetic flux, $\Phi$, is coupled to the arrays. Families of $d c I V C$ for different values of $\Phi$ were measured for the arrays by a 4 pointcontact method. The applied $\Phi$ excites multiple flux-flow resonances in the array's IVCs. In the proximity of the symmetric $100 \mathrm{JJ}$-arrays, JJ EM detectors were patterned (see Fig. 1) and their IVC's could be measured independently. The JJ detectors consisting of a single JJ $9 \mu \mathrm{m}$ wide were inductively and capacitively coupled to the $100 \mathrm{JJ}$-arrays. When the array is biased at or around the resonance powerful EM is emitted that induces Shapiro steps on the IVCs of the $\mathrm{JJ}$ detectors.

A typical data set at a temperature $\mathrm{T}=45 \mathrm{~K}$ for the symmetric $100 \mathrm{JJ}$-array consisting of a family of 203 IVCs (a 1,000 points each) measured for different values of Ів changed in steps of $5 \mu \mathrm{A}$ in the range $(-0.41,0.6) \mathrm{mA}$ is shown in Figs. $2 \mathrm{a}$ and $2 \mathrm{~b}$. In Fig. 2a, 13 consecutive IVCs and their corresponding $\mathrm{dI} / \mathrm{dV}(\mathrm{V})$ curves are plotted, respectively, showing the variation of the flux- flow resonance $m=1$ voltage's peak position $V_{\text {res }}$ with $B$. From the $\mathrm{dI} / \mathrm{dV}(\mathrm{V})$ curves, $\mathrm{V}_{\text {res }}\left(\mathrm{I}_{\mathrm{B}}\right)$ of the $\mathrm{m}=1$ resonance was extracted (red squares in Fig. 2b). $\mathrm{V}_{\text {res }}\left(\mathrm{I}_{\mathrm{B}}\right)$ shows a periodicity with $\mathrm{I}_{\mathrm{B}}$ of $0.235 \mathrm{~mA}$ that corresponds exactly to an additional $\Phi_{0}$ in each superconducting loop area Aloop separating the $J J_{\mathrm{S}}$ as determined from calibration measurements of periodic voltage oscillations of YBCO-SQUIDs with identical loop areas patterned separately ${ }^{23}$. This physical interpretation for the period of $\mathrm{V}_{\mathrm{res}}\left(\mathrm{I}_{\mathrm{B}}\right)$ curve could also be independently verified by analysing $\mathrm{V}\left(\mathrm{I}_{\mathrm{B}}\right)$ curves taken at different current biases that are 
expected to show a similar $\Phi_{0}$-periodicity (see Fig. 5 in the Supplementary Material) in agreement with the theoretical calculations for such $J J$-arrays ${ }^{24,25}$. Indeed, at temperatures close to $T_{c}=49 \mathrm{~K}$ we are in the low-inductance limit and the magnetic self-field effects due to the superconducting currents in the loops can be neglected. In this case $\mathrm{V}\left(\mathrm{I}_{\mathrm{B}}\right)$ consists of a series of maxima periodically distributed with І $_{\text {B }}$ The periodicity in $I_{B}$ corresponds to one additional flux quantum $\Phi_{0}$ in each superconducting loop separating the JJs and is similar to a diffraction pattern of an optical grating consisting of multiple slits.

A 3D map of dI/dV(V, Iв) (see Fig. 3a) exploits the entire 203 IVC $\times 1,000$ data points matrix measured. Such 3D maps of flux-flow resonances are very useful in representing the complexity/richness of flux-flow dynamics involving multiple flux-flow resonances $m=1-4$, their mutual interference/splitting and $\Phi_{0}$-periodicity with $B$. They unveil a remarkable continuity of flux-flow resonances with multiple flux-flow resonances coexisting in the entire $\left(\mathrm{V}, \mathrm{I}_{\mathrm{B}}\right)$ range investigated. This is in striking contrast to previously reported cases of either single long $J J^{1,7}$ or $J J$-arrays ${ }^{16-20,22}$ made of low-temperature superconductors where fluxflow resonances are short-lived while varying $B$. For positive current biases, resonance $m=1$ split into two, $\mathrm{m}=1$ and $\mathrm{m}=2$, at $\mathrm{V}=0.04 \mathrm{mV}$. Initially the voltage position of the $\mathrm{m}=1$ resonance linearly increases with increasing $B$ up to $\mathrm{V}=1.6 \mathrm{mV}$, but then slightly decreases linearly with $B$ at the same rate (see also Fig. $2 b$ ). The voltage position of the $\mathrm{m}=2$ resonance linearly decreases with increasing $B$. This cycle repeats itself with a $\Phi_{0}$-periodicity. As a result, a peculiar pattern of small and large triangles overlapping each other is formed (in yellow and light-blue colours). For negative current biases the pattern of the flux-flow resonance dynamics is similar but lacks both reflection symmetry with respect to $V=0$ axis and point symmetry with respect to $B=0$ as it is shifted on the $B$ field axis by a value corresponding to a fraction of $\Phi_{0}$. This shift is due to the presence of a remnant non-zero flux associated with the unshielded earth magnetic field in the array/cryostat. The height of $m=1$, 2 resonances, and consequently, the power of emitted EM-radiation, both monotonically decrease with increasing voltage. As $\mathrm{V}_{\text {res }}$ of various flux-flow resonances changes with $B$ in the range $(-0.3,0.3) \mathrm{mV}$ the frequency, $f=\mathrm{V} / \Phi_{0}$, of the emitted EM-radiation changes in the range $(0,0.145) \mathrm{THz}$.

Flux-flow resonances in the temperature range (77-89) K were previously observed ${ }^{21}$ also in the asymmetric $440 \mathrm{JJ}$-arrays considered again here. Here we demonstrate their continuity with a changing magnetic field suggesting an ideal tunability, with no gaps in the 
frequency range of the emitted EM. Again, such stability of flux-flow resonances over a wide range of both relevant parameters B and V in HTS JJ-arrays operating at $77 \mathrm{~K}$ and above is in sharp contrast to LTS JJ-arrays ${ }^{16-20,} 22$ operating at $4.2 \mathrm{~K}$, representing a considerable advantage in applications since it improves the ability to tune the emitted EM radiation with B. A 3D map for the strength of flux-flow resonances $\mathrm{dI} / \mathrm{dV}\left(\mathrm{V}, \mathrm{I}_{\text {в }}\right.$ (see Fig. 3b) in this case based on 302 IVCs measured at $84 \mathrm{~K}$ reveals an enriched dynamics with an intense interplay/superposition of multiple and sharper flux-flow resonances relative to the case of symmetric arrays. Moreover, in asymmetric arrays up to four sharp resonant modes could be present simultaneously for some $B$ field values while their intensity does not decrease with increasing voltage (compare Figs. $3 a$ and $3 b$ ). This suggests an enhanced power for the emitted EM-radiation throughout the entire $\left(\mathrm{V}, \mathrm{I}_{\mathrm{B}}\right)$ range of operation investigated estimated to be of the order of up to $100 \mathrm{nW}^{21}$. A direct comparison of the $\mathrm{dI} / \mathrm{dV}(\mathrm{V})$ curves of the symmetric $100 \mathrm{JJ}$-array and the asymmetric $440 \mathrm{JJ}$-array (see also Fig. $3 \mathrm{~b}$ in $^{21}$ ) is presented in Fig. 4a. Roughly speaking the dynamic conductance $d \mathrm{I} / \mathrm{dV}_{\text {res }}$ at the peak of flux-flow resonances in $440 \mathrm{JJ}$-arrays are about 10 times larger in amplitude and occur at about 10 times lower voltages. Considering that the EM radiation linewidths $\Delta \mathrm{f}$ in the flux-flow regime is given by ${ }^{26}$

$$
\Delta f=\frac{\pi k_{B} T}{\Phi_{0}^{2}} \frac{1}{R(d I / d V)^{2}}
$$

with $\mathrm{k}_{\mathrm{B}}$ being the Boltzmann constant and $\mathrm{R}$ the array's resistance, one can estimate that the asymmetric $440 \mathrm{JJ}$-array has a linewidth $\Delta \mathrm{f}=0.78 \mathrm{MHz}$ at an $\mathrm{EM}$ radiation frequency $f=\mathrm{V}_{\text {res }} / \Phi_{0}$ of $5 \mathrm{GHz}$, which is about 240 times narrower than for the symmetric 100-JJ at 36 $\mathrm{GHz}$. Here the following values for the $\left(\mathrm{R}, \mathrm{T}, \mathrm{dI} / \mathrm{dV}_{\text {res }}, \mathrm{V}_{\text {res }}\right)$ parameters were determined: ( 8 $\left.\mathrm{m} \Omega, 45 \mathrm{~K}, 17.5 \Omega^{-1}, 75 \mu \mathrm{V}\right)$ for the symmetric $100-\mathrm{JJ}$ array and $\left(15 \mathrm{~m} \Omega, 84 \mathrm{~K}, 270 \Omega^{-1}, 10.5\right.$ $\mu \mathrm{V}$ ) for the asymmetric 440-JJ array. The relative linewidth $\Delta f / f$ is therefore 33 times better for the asymmetric design.

To detect the emitted EM-radiation we biased the $100 \mathrm{JJ}$-array at the peak of the resonance $m=1$ where emitted power is maximum while independently recording the IVCs of single $J J s$ used as detectors ${ }^{1,2,27}$ and observing the EM-induced Shapiro steps ${ }^{11}$. As we change $B$ (or equivalently, $\left.\mathrm{I}_{\mathrm{B}}\right), \mathrm{V}_{\text {res }}\left(\mathrm{I}_{\mathrm{B}}\right)$ changes too, and each time we have re-adjusted the array's bias current so that it corresponded to the peak in the $\mathrm{dI} / \mathrm{dV}(\mathrm{V})$ plot and then again have recorded an IVC of the $J J$-detector. Typically, the EM-radiation emitted by the $100 \mathrm{JJ}$ array induces between one and three Shapiro steps on the IVC's of the JJ-detector. We have 
determined the frequency of the emitted EM-radiation from the voltage position of the first Shapiro step via the Josephson relation $f=\mathrm{V} / \Phi_{0}$. For accurate readings of $f, \mathrm{dI} / \mathrm{dV}(\mathrm{V})$ curves of the $J J$-detector were used instead of the IVCs. Using this procedure $f\left(\mathrm{I}_{\mathrm{B}}\right)$ could be plotted (blue dots in Fig. 2b). For voltages larger than about $100 \mu \mathrm{V}$ the emitted EM power was insufficient to induce Shapiro steps in our JJ-detectors and no readings could be made. This can be well understood considering that the power of the emitted radiation is proportional to the height of the flux-flow resonance $m=1$ which, within each $\Phi_{0}$ period, monotonically decreases with increasing the voltage of the resonance peak. The detection of EM-radiation can be drastically improved in practice as discussed later. In our case one has a rather weak coupling and a significant impedance mismatching between the $100 \mathrm{JJ}$-arrays as the source of the EM and the JJ-detectors. Two examples are shown in Fig. $4 \mathrm{~b}$ where the $\mathrm{dI} / \mathrm{dV}(\mathrm{V})$ of the $100 \mathrm{JJ}$-array, as well as, the corresponding induced Shapiro steps on the detector's $\mathrm{dI} / \mathrm{dV}(\mathrm{V})$ are plotted (see insets) with $\mathrm{I}_{\mathrm{B}}$ changed from $-0.18 \mathrm{~mA}$ to $-0.12 \mathrm{~mA}$. Accordingly, $\mathrm{V}_{\text {res }}\left(\mathrm{I}_{\mathrm{B}}\right)$ changes from $27 \mu \mathrm{V}$ to $75 \mu \mathrm{V}$, while the corresponding voltage readings from the Shapiro steps are $24 \mu \mathrm{V}$ and $65 \mu \mathrm{V}$, respectively (attributed to emitted EM-radiation of frequency $f=\mathrm{V} / \Phi_{0}$ of $11.6 \mathrm{GHz}$ and $31.5 \mathrm{GHz}$, respectively). The agreement between the voltage recordings $\mathrm{V}_{\text {res }}\left(\mathrm{I}_{\mathrm{B}}\right)$ from the $J J$-array (red squares in Fig. 2b) and the frequencies readings $f\left(\mathrm{I}_{\mathrm{B}}\right)$ from the $J J$-detector (blue dots in Fig. $2 \mathrm{~b}$ ) is remarkable. This agreement means that one can precisely measure $\Phi$ (or $B$ ) applied to the $100 \mathrm{JJ}$-array wirelessly, from a distance, without directly measuring it locally, by reading the frequency of the emitted EM-radiation. A unique feature is that $f$ of the emitted EM-radiation has a $\Phi_{0}$-periodicity with the applied flux $\Phi$. Superconducting loops with very large area require a very small change in $B$ to achieve a complete $\Phi_{0}$-periodical oscillation of $f$. This can be exploited to develop an ultra-sensitive remote flux-to-frequency magnetometer $f(\Phi)$ based on the detected frequency of the emitted EM-radiation. To operate it in various applications an on-chip compact dc battery would be required, but no direct wire connections to a voltmeter or any other read-out scheme would be necessary, making it practical for remote operations. In our proof-of-concept case the flux-tofrequency conversion resolution for the $100 \mathrm{JJ}$-array is about $50 \mathrm{GHz} / \Phi_{0}$. This resolution can easily be translated into an ultra-high field-to-frequency sensitivity $f(B)$ by implementing advanced flux-to-field conversion and read-out techniques routinely used in SQUID electronics ${ }^{14}$ (including flux-locked loop technique, single layer/multilayers flux transformers, flux-focusers, etc). Thus, analogue to the case of SQUIDs ${ }^{14}$ the signal to be measured can be 
more efficiently coupled to the JJ-arrays directly or inductively, using washer-like fluxfocusers for the SQUID loops and flux transformers ${ }^{28}$. Of particular relevance here is the implementation of narrow flux-focusers as used in the development of SQUID-arrays operating flux-coherently ${ }^{23}$. The field sensitivity $f(B)$ also strongly depends on the sensitivity by which the EM radiation is detected. Such calculations are beyond the scope of this report, as here, our main purpose is to present a valid qualitative proof of concept. Indeed, the EMradiation detection can be significantly improved by implementing superior methods, such as on-chip detection via direct coupling of the $J J$-detectors to the $J J$-arrays via coplanar microstrip resonators ${ }^{1,27}$, balanced/harmonic mixers ${ }^{3}$, or off-chip heterodyne detection technique ${ }^{27}$ and quasi-optical Schottky diodes ${ }^{29}$ operating at room or cryogenic temperatures. Another convenient room-temperature detection scheme ${ }^{22}$ involves a coplanar waveguide and a low-noise high gain amplifier.

In conclusion, we detected on-chip flux-flow-induced EM-radiation at $45 \mathrm{~K}$ from symmetric $100 \mathrm{JJ}$-arrays made of YBCO whose frequency could be tuned in the range (1-50) $\mathrm{GHz}$ periodically by an applied magnetic flux with a $\Phi_{\theta}$-periodicity. We have therefore demonstrated the principle of operation of a remote flux-to-frequency magnetometer $f(\Phi)$ based on the detected frequency of the emitted EM-radiation. Asymmetric $440 \mathrm{JJ}$-arrays operating in the range (77-89) $\mathrm{K}$ showed a superior performance with an enhanced flux-flow dynamics, sharper multiple resonances suggesting a narrower emission-linewidth and an increased emitted EM-radiation power of about $100 \mathrm{nW}$. Such asymmetric arrays are therefore promising elements for the development of sub-terahertz integrated-receivers to be used in radio-astronomy and atmospheric science at higher, more practical, temperatures than are currently operated at $4.2 \mathrm{~K}$. Numerical simulations performed are in qualitative agreement with the measurements (see Supplementary material).

The research at Loughborough University (LU) was partially supported by the Higher Education Innovation Fund (HEIF) and Loughborough Enterprise Projects Group (EPG), EPG 101, 2017, LU code: S11519.

\section{SUPPLEMENTARY MATERIAL}

See supplementary material for details related to JJ-array fabrication, measurements and numerical simulations. 


\section{DATA AVAILABILITY}

The data that support the findings of this study are available from the corresponding author upon reasonable request.

\section{REFERENCES}

1. T. Nagatsuma, K. Enpuku, and F. Irie, J. Appl. Phys. 54, 3302 (1983).

2. S.P. Benz and D.J Burroughs, Appl. Phys. Lett. 58, 2162 (1991).

3. P. A. A. Booi, and S. P. Benz, Appl. Phys. Lett. 64, 2163-2165 (1994).

4. V. P. Koshelets, S. Shitov, L.V. Fillippenko, A. M. Baryshev, Appl. Phys. Lett. 68, 1273 (1996)

5. Y. M. Zhang, D. Winkler, P.-Å. Nilsson, and T. Claeson, Phys. Rev. B 51, 8684(R) (1995).

6. R.E. Eck, D.J. Scalapino and B. N. Taylor, Phys. Rev. Lett.13, 15-8 (1964).

7. S.N. Erne and R.D. Parmentier, J. Appl. Phys. 51, 5025 (1980).

8. V. P. Koshelets and S. V. Shitov, Supercond. Sci. Technol. 13R53 (2000).

9. V. P. Koshelets, A. B. Ermakov, L. V. Filippenko, A. V. Khudchenko, O. S. Kiselev, A. S. Sobolev, M. Y. Torgashin, P. A. Yagoubov, R. W. M. Hoogeveen, and W. Wild, IEEE, Trans. Appl. Supercond. 17, 336 (2007).

10. G. D. Lange, M. Birk, D. Boersma, J. Dercksen, P. Dmitriev, A. B. Ermakov, L. V. Filippenko, H. Golstein, R. W. M. Hoogeveen, L. D Jong, et al., Supercond. Sci. Technol. 23, 045016 (2010).

11. S. Shapiro, Physical Review Letters 11, 80 (1963).

12. B.S. Deaver and W.M Fairbank, Phys. Rev. Lett.7, 43-46 (1961).

13. R. Doll and M. Naebauer, Phys. Rev. Lett. 7, 51-52 (1961).

14. The SQUID Handbook, Vol. 1, Eds. J. Clarke and A. I. Braginsky, 2004.

15. M. Cirillo, S. Pace and B. Savo, IEEE Trans. Magn. 19, 1021 (1983).

16. V.A. Oboznov and A.V. Ustinov, Phys. Lett. A 139, 481-484 (1989).

17. H. S. J. Van der Zant, T. P. Orlando, S. Watanabe, and S. H. Strogatz, Phys. Rev. Lett. 74, 174-177 (1994).

18. H.S.J. Zant and T.P. Orlando, J. Appl. Phys. 76, 7606-7612 (1994).

19. A. E. Duwel, S. Watanabe, E. Tria's, T. P. Orlando, H. S. J. Zant, and S. H. Strogatz, J. Appl. Phys. 82, 4661-4668 (1997). 
20. A. V. Ustinov, M. Cirillo, Britt H. Larsen, V. A. Oboznov, P. Carelli, and G. Rotoli, Phys. Rev. B 51, 3081-3091 (1995).

21. B. Chesca, D. John, and C. J. Mellor, Supercond. Sci. Technol. 27, 085015 (2014).

22. M. Lucci, D. Badoni, V. Merlo, I. Ottaviani, G. Salina, and M. Cirillo, A. V. Ustinov and D. Winkler, Phys. Rev. Lett. 115, 107002 (2015).

23. B. Chesca, D. John, and C. J. Mellor, Appl. Phys. Lett. 107, 162602 (2015);

24. J.H. Miller, G. H. Gunaratne, J. Huang, and T.D. Golding, Appl. Phys. Lett. 59, 3330 (1991).

25. M. Lucci, V. Merlo, I. Ottaviani, M. Cirillo, D. Badoni, V. Campanari, G. Salina, J. G. Caputo, and L. Loukitch, Appl. Phys. Lett. 113, 192601 (2018).

26. E. Joergensen, V. P. Koshelets, R. Monaco, J. Mygind, M. R. Samuelsen, and M. Salerno, Phys. Rev. Lett. 49, 1093 (1982).

27. K. Lee, Iguchi and K. Constantinian, IEEE Trans. Appl. Supercond. 9, 4333-4336 (1999); Physica C 320, 65 (1999).

28. M. B. Ketchen, and J. M. Jaycox, Appl. Phys. Lett. 40, 736 (1982).

29. L.Liu, J. L. Hesler, H. Xu, A. W. Lichtenberger, and R. M. Weikle, IEEE Microw. Wireless Compon. Lett., 20, 504-506, (2010). 
Figure captions

Fig. 1. Optical micrograph of the device and its electrical equivalent circuit. a) Optical micrograph showing a $100 J J$-array coupled to a single $J J$ EM-radiation detector. The $J J$ s are formed across the bicrystal grain boundary shown with dotted line. A magnetic field $B$ is applied perpendicular to the planar structure. When biased with a dc current I magnetic vortices start to flow across the $100 \mathrm{JJ}$-array in the direction show by the bold horizontal arrow. When vortices exit the array EM-radiation is emitted that excite Shapiro steps on the current-voltage characteristic $\mathrm{ID}_{\mathrm{D}}-\mathrm{V}_{\mathrm{D}}$ of the $J J$ detector inductively and capacitively (C) coupled to the array. b) Schematic view of the equivalent circuit.

Fig. 2. Experimental data set at a temperature of $45 \mathrm{~K}$. a) 13 consecutive IVC's of the $100 \mathrm{JJ}$ array measured for different applied $B$ fields (or equivalently, fluxes $\Phi$ ) with Iв changed in steps of $5 \mu \mathrm{A}$ showing the flux-flow resonance $\mathrm{m}=1$. Insets: $\mathrm{dI} / \mathrm{dV}(\mathrm{V})$ for negative voltages (left) and positive voltages (right). The horizontal arrows show the shifts of the peak voltage $\mathrm{V}_{\text {res }}$ of $\mathrm{m}=1$ to lower (higher) values with $B$. The central peak around $\mathrm{V}=0$ is due to the array's Josephson critical current. b) Red squares: $\mathrm{V}_{\text {res }}\left(\mathrm{I}_{\mathrm{B}}\right)$ of the $\mathrm{m}=1$ resonance extracted from a family of $203 \mathrm{dI} / \mathrm{dV}(\mathrm{V})$ curves of the $100 \mathrm{JJ}$-array. Blue dots: frequency $f$ of the emitted EM-radiation calculated from the voltage position of the first Shapiro step induced on the $J J$-detector versus $B$ field current $\mathrm{I}_{\text {в. }} f$ and $\mathrm{V}$ are related via the $a c$ Josephson equation $f=\mathrm{V} / \Phi_{0}$. IB was changed in steps of $20 \mu \mathrm{A}$ in the range $(-0.4,0.4) \mathrm{mA}$ in this case.

Fig. 3. 3D plots of $\mathrm{dI} / \mathrm{dV}\left(\mathrm{V}, \mathrm{I}_{\mathrm{B}}\right)$. The rich network of yellow, light-blue lines crossing each other and showing a $\Phi_{0}$-periodicity are due to multiple flux-flow resonances. The yellow vertical bands centred at $\mathrm{V}=0$ are due to the array's Josephson junction's critical current. a) Extracted from a family of 203 IVC's measured for a symmetric $100 \mathrm{JJ}$-array for different values of the $B$ field current Ів. Ів was changed in steps of $5 \mu \mathrm{A}$ in the range $(-0.41,0.6) \mathrm{mA}$. b) Extracted from a family of 301 IVC's measured for an asymmetric $440 \mathrm{JJ}$-array for different values of the $B$ field current $\mathrm{I}_{\mathrm{B}}$. Ів was changed in steps of $15 \mu \mathrm{A}$ in the range $(-2.25$, 
2.25) $\mathrm{mA}$. To allow the use of the same scale the $\mathrm{dI} / \mathrm{dV}$ values have been divided by 10 in this case.

Fig. 4. a) A direct comparison of typical dI/dV(V) curves for the $100 \mathrm{JJ}$-array at $45 \mathrm{~K}$ and the $440 \mathrm{JJ}$-array at $84 \mathrm{~K}$. Note that for the $440 \mathrm{JJ}$-array case the scale for $\mathrm{V}(\mathrm{dI} / \mathrm{dV})$ is 10 times smaller (larger). b) $\mathrm{d} / / \mathrm{dV}(\mathrm{V})$ curves of the $100 \mathrm{JJ}$-array at $45 \mathrm{~K}$ for two different values of $B$ corresponding to Iв $-0.18 \mathrm{~mA}$ and $-0.12 \mathrm{~mA}$ (shown also in (a)). The peak voltage of the $\mathrm{m}=1$ flux-flow resonance changes from $27 \mu \mathrm{V}$ to $75 \mu \mathrm{V}$. Each time we biased the $100 \mathrm{JJ}$-array at the resonance peak the emitted EM-radiation is maximum and can be detected on-chip by measuring the frequency of Shapiro steps induced in the $\mathrm{dI} / \mathrm{dV}(\mathrm{V})$ of the JJ detector (see insets). The voltage position of the first induced Shapiro step for these two B values are 24 $\mu \mathrm{V}$ and $65 \mu \mathrm{V}$, respectively. 
Figure 1

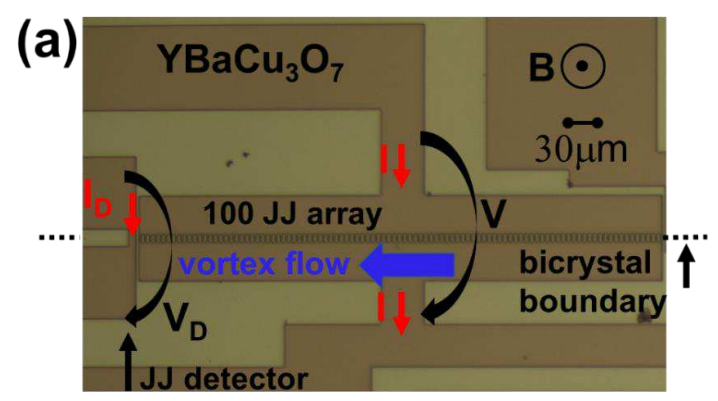

(b) 
Figure 2
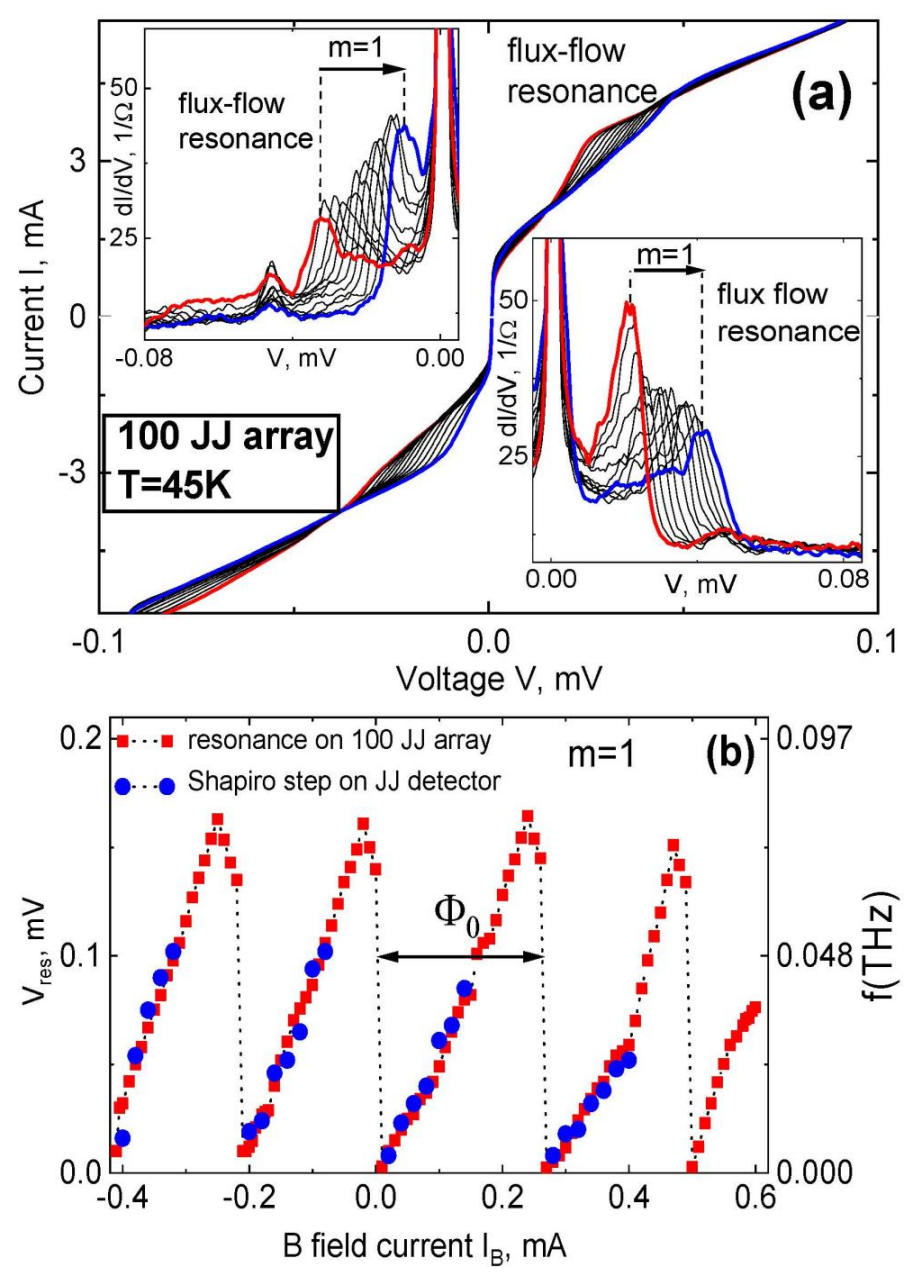
Figure 3

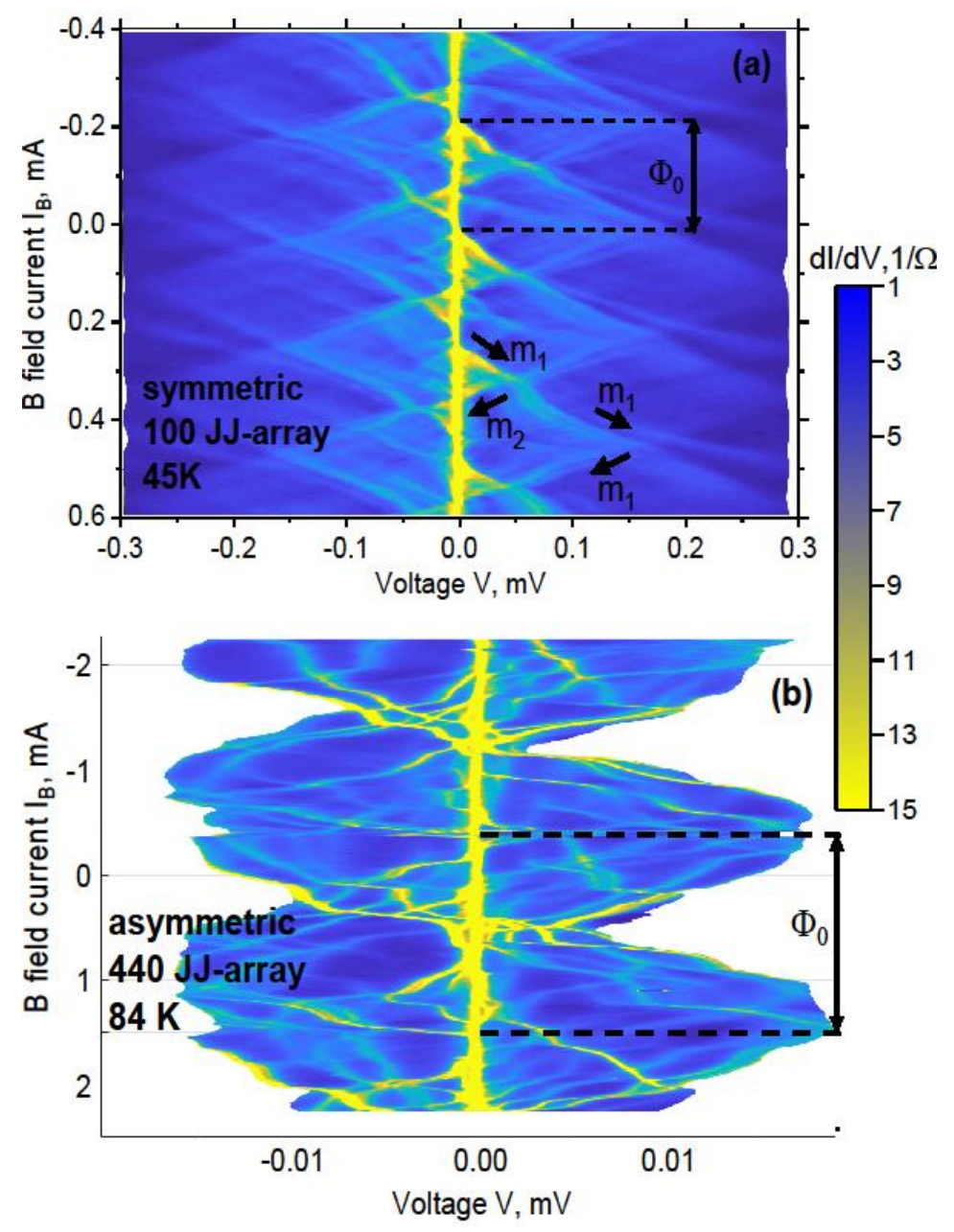


Figure 4 

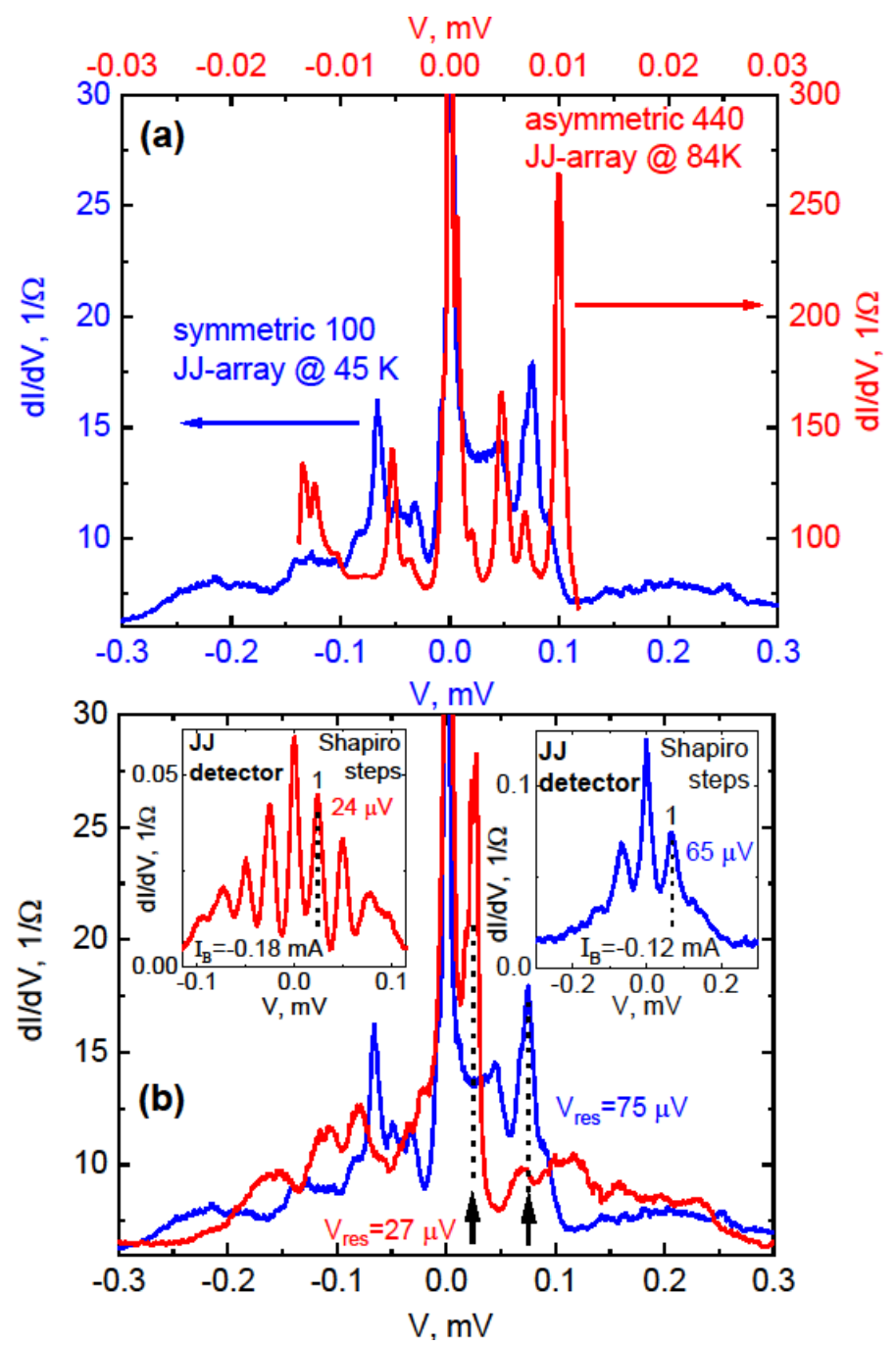
This is the author's peer reviewed, accepted manuscript. However, the online version of record will be different from this version once it has been copyedited and typeset.

(a)

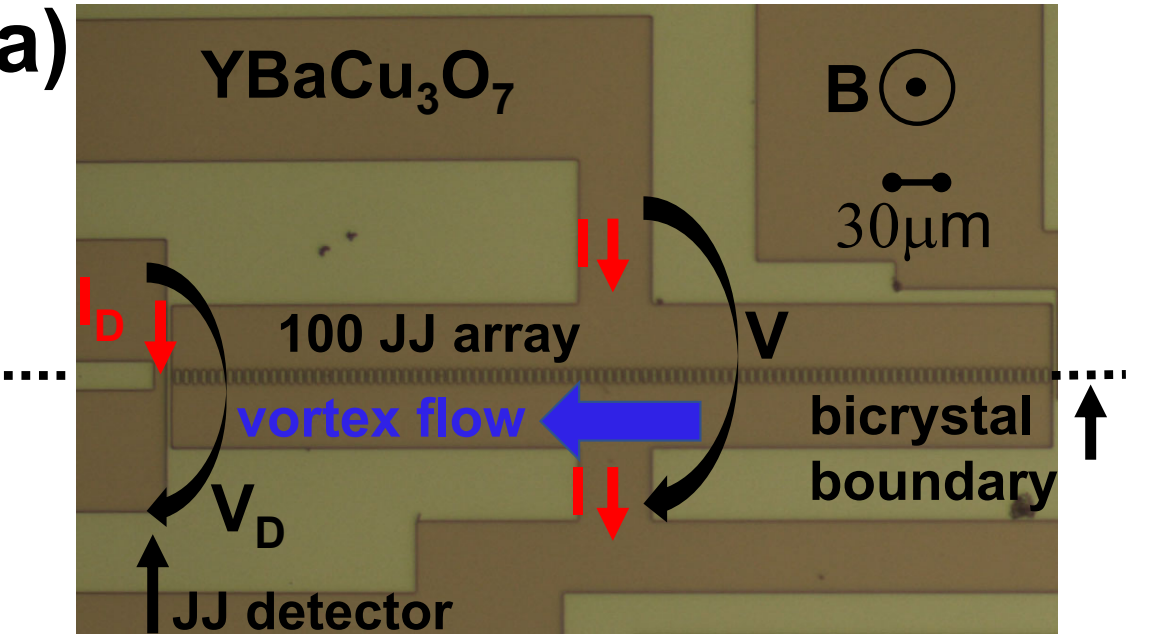

(b)

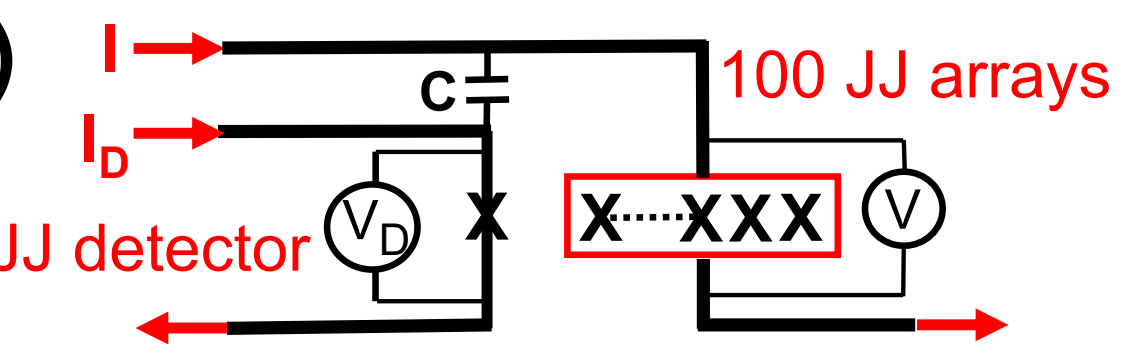



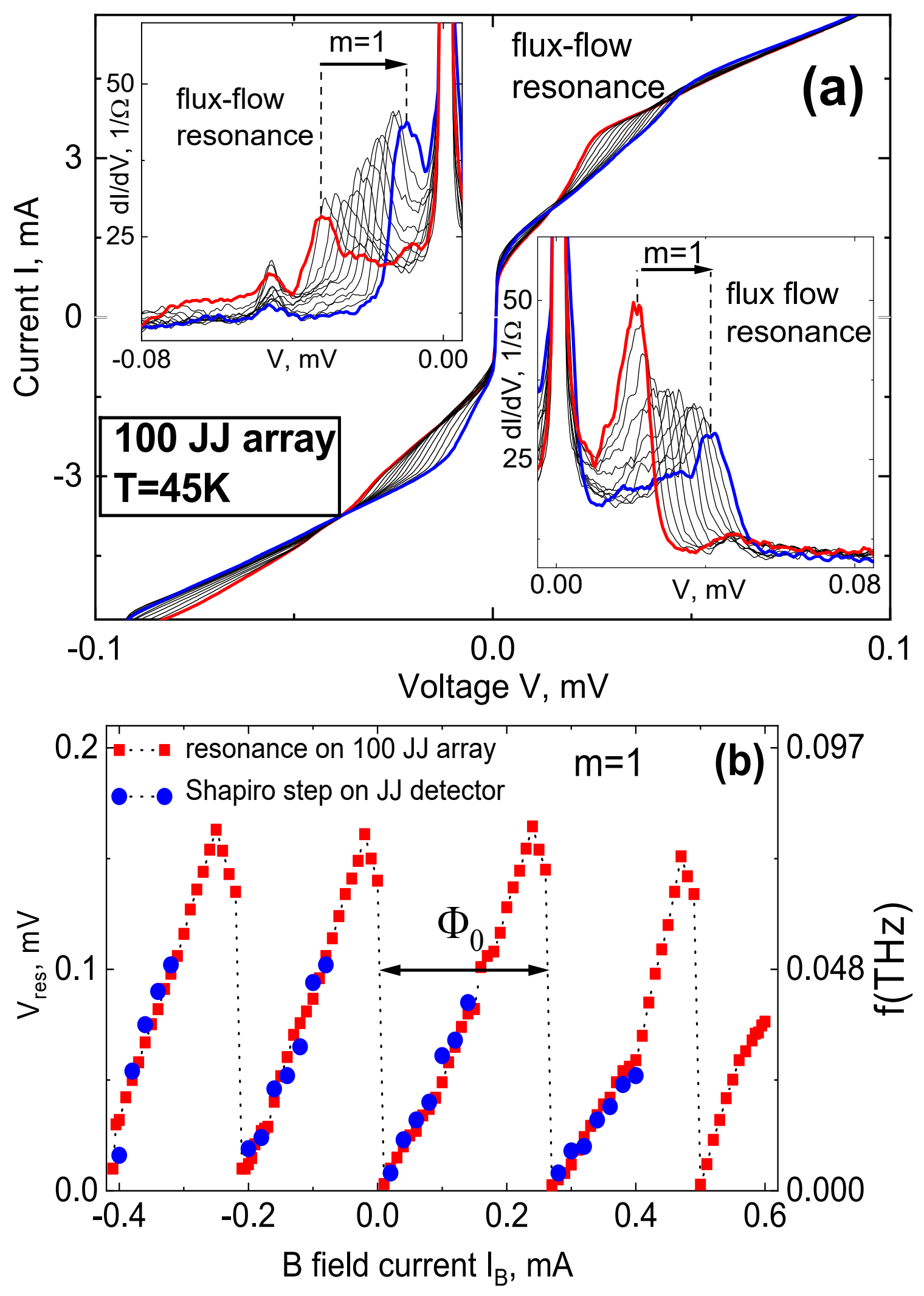


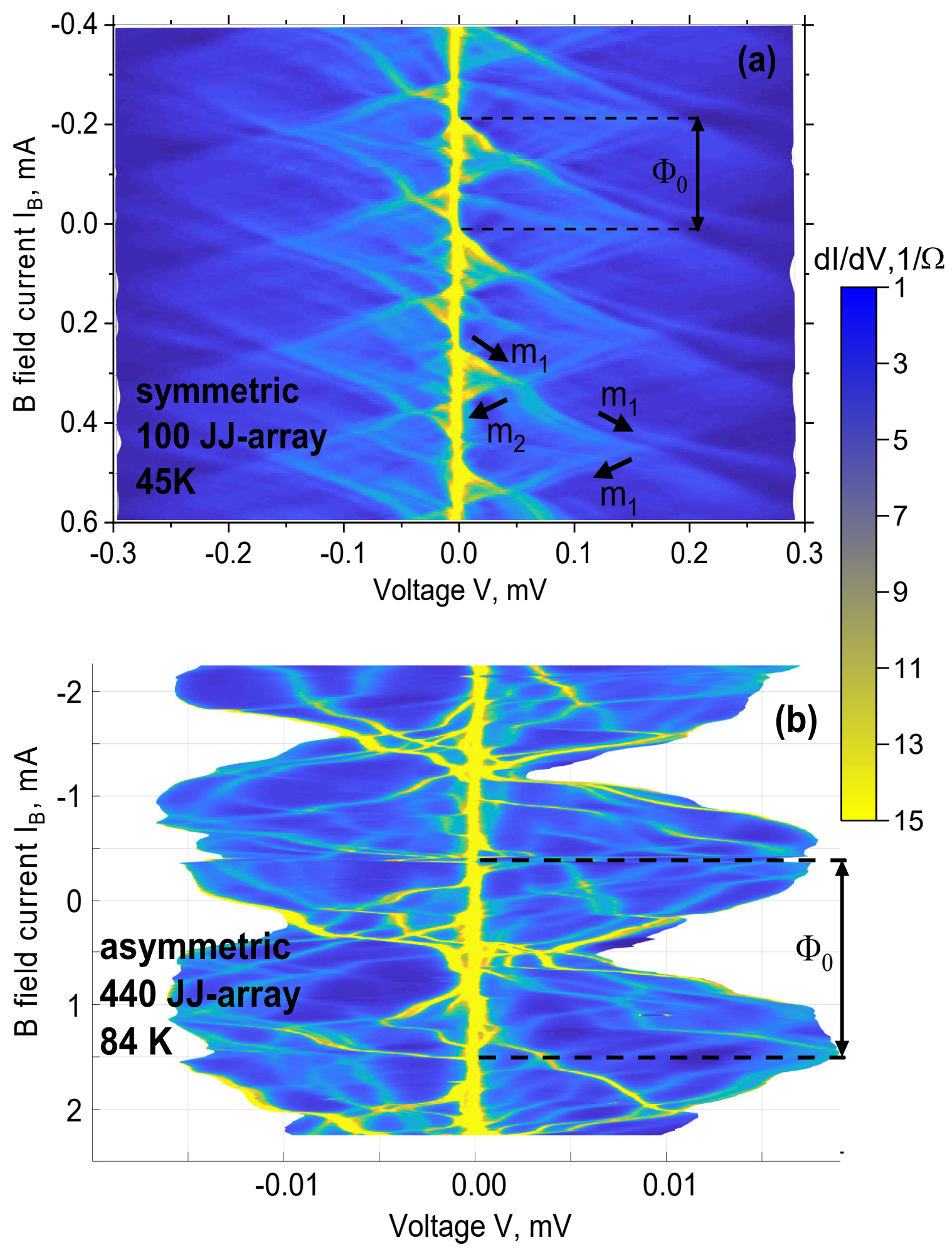




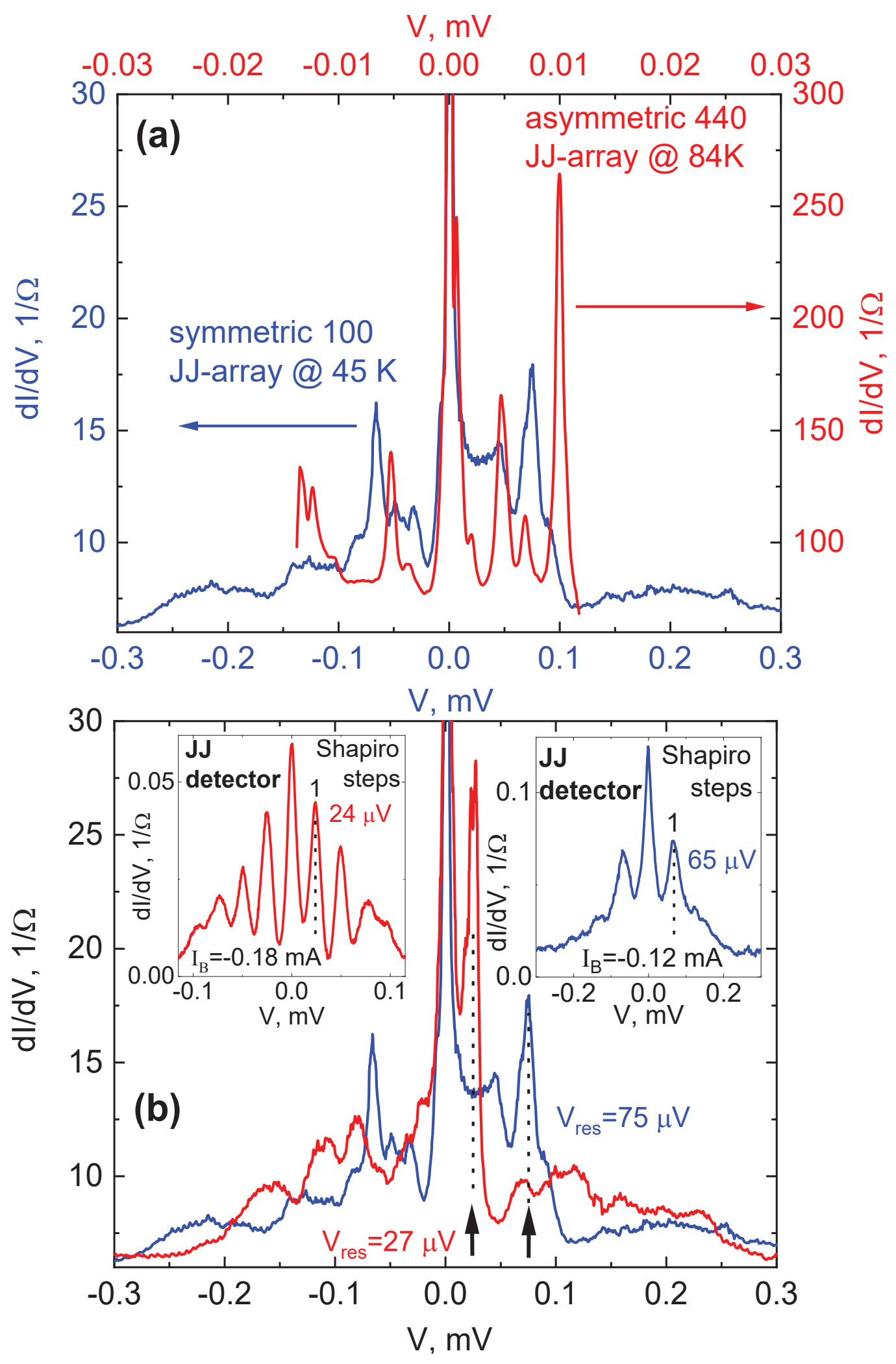

